(Aus dem pathologisch-anatomischen Institut am Friedrichstädter Krankenhaus zu Dresden, Direktor: Geh.-Rat Prof. Dr. Schmorl).

\title{
Zur Kenntnis der Strangentartung des Rückenmarks bei perniziöser Anämie.
}

\author{
Von \\ Dr. med. F. Thoenes.
}

Nachdem Lichtheim im Jahre 1887 zum ersten Male auf die enge Verknüpfung besonderer Rückenmarkserkrankungen mit dem Symptomenkomplexe der perniziösen Anämie hingewiesen und zwei einschlägige Fälle mit anatomischem Befunde veröffentlicht hatte, blieb das Interesse der Neuropathologie stetig dem Gegenstand zugewendet. Schon im Jahre 1884 hatte Leichtenstern zweimal Rückenmarkserkrankungen hei perniziöser Anämie beobachtet, die er indessen unter das Bild der Tabes dorsalis einreihen zu müssen geglaubt hatte. Lichtheim und seinem Schüler Minnich (1892) blieb es vorbehalten, durch genaue klinische Beobachtung und eingebende anatomische Untersuchungen, die sich ins Gesamt auf 6 Fälle erstreckten, jene als etwas Besonderes zu erkennen, und das damit neu bekannt gewordenc Kranlsheitsbild gegen die Tabes dorsalis abzugrenzen. Im Jahre 1891 veröffentlichte auch v. Noorden einen einschlägigen Fall.

In allen Fällen handelte es sich bei, sowohl in vivo, wie in autopsia für perniziöse Anämie typischen Organbefunde, um mehr oder weniger akut verlaufende Rückenmarkserkrankungen, deren kürzeste Dauer auf 2, deren längste auf 9 Monate angegeben wurde. Die klinischen Erscheinungen waren sehr mannigfaltig. Während im Beginn meistens Parästhesien und motorische Schwäche, selten auch Schmerzen in den unteren Extremitäten zu bemerken waren, stellten sich mit dem Fortschireiten der Krankheit objektiv nachweisbare Sensibilitätsstörungen ein. Auch die Temperaturempfindung konnte gestört sein. Meist wurde auch Ataxie beobachtet, der sich bald eine stärkere $A b$ nahme der groben Kraft bei Frhaltenbleiben oder allmählichem Schwinden der Patellarreflexe hinzugesellte, oder es waren spastisch paretische 
Symptome mit starker Steigerung der Sehnenreflexe und wohl auch klonische Zuckungen der Muskulatur nachzuweisen. In einem von den bis dahin genau beobachteten 9 Fällen wurde anch Pupillenstarre gefunden. Blasen- und Mastdarmstörungen stellten sich meist erst gegen Ende des Lehens ein, nachdem die Erkrankung häufig auch die oberen Extremitäten in minderer Stärke ergriffen hatte.

Anatomisch waren immer Degenerationen im Bereich der Hinterstränge nachweisbar, die sich im Hals- und oberen Brustmark am stärksten ausprägten und dort im Bereiche der Gollschen Stränge stellenweise systematischen Charakter annahmen. In den unteren Teilen des Marks war die Hinterstrangsdegeneration immer mehr oder weniger fleckig-herdförmig, besonders in den Keilsträngen des Lendenmarks, wo die mittleren Wurzelzonen der Prädilektionssitz der Veränderungen zu sein schien. Ganz unregelmäßige fleckig-herdförmige Veränderungen wurden bei den meisten Fällen auch in den Vorder- und Seitensträngen gefunden, während die Wurzeleintrittszone, Lissauersche Randzone, sowie die graue Marksubstanz ganz intakt waren oder nur geringfügige Veränderungen aufwiesen. Die peripheren. Wurzeln waren nie verändert. Fast überall wurde beobachtet, daß sich die herdförmigen Degenerationen dicht an die mit den Septen einstrahlenden Gefäße anschloșsen, so daß immer im Zentrum eines Herdes ein Gefäß zu liegen kam. Diese selbst , boten oft nur auffällig wenig Veränderungen dar" Minnich beobachtete eine "Verdickung der Media" und „Wucherung der Kerne in der adventitiellen Lymphscheide", während v. Noorden nur jere Rundzellinfiltration nachzuweisen vermag. Minnich fand die Neuroglia, besonders in den Hintersträngen oft deutlich gewuchert mit entsprechender Kernvermehrung, stellenweise aber, rornehmlich in der Peripherie der Herde zahlreiche Lücken. Noorden lingegen meint, daß die Nervensubstanz einfach ïberall geschwunden sei, so daß das bindegewebige Gerïst ale netzförmiges Maschenwerk zuriickblieb.

Die Deutung der vorliegenden Befunde, die eine auffällige Inkongurrenz mit den klinischen Symptomen darbot, w urde durch zwei Tatsachen kompliziert. Lich theim hatte ähnliche Befunde an Rürkenmarken bei anderen Allgemeinerkrankungen, z. B. Diabetes erhoben und Minnich konnte Rückenmarksveränderungen auch in solchen Fällen perniziöser Anämie nachweisen, die intra vitam keine klinischen Symptome solcher gezeigt hatten.

Das Hauptaugenmerk galt der Pathogenese des Prozesses und seiner Ätiologie. Sowohl Lichtheim wie Minnich kamen zu 
der Ansicht, es möchte sich bei den Rückenmarksveränderungen wohl um eine Folge der Anämie handeln können im Sinne einer Autointoxikation. Zugleich aber weisen sie anf die Ähnlichkeit der Veränderungen mit denen, die bei Ergotismus, Pellagra und anderen Intoxikationen beobachtet werden, hin, die es wohl möglich erscheinen lassen, daß Anämie und Rückenmarkserkrankang Folge einer noch unbekannten gemeinsamen Noxe sein kann. So erachtet auch v. Noorden Anämie und Rückenmarkserkrankung als koordinierte Erscheinungen. Diese selbst deutet Minnich als bedingt durch primäre Degenerationsherde, die über den ganzen Bercich des Riickenmarks regellos rerstreut, besonders im Bereich der Hinter stränge, und da wieder mit Vorliebe in der mittleren Wurzelzone, hauptsächlich des Lendenmarks lokalisiert sind. Diese verursachen durch die Zerstörung der in den mittleren Wurzelzonen nach oben konvergierenden langen Hinterwurzelfasern eine sekundäre Degeneration der Gollschen Stränge. Die herdförmigen Degenerationen, die ebenso in den Vorder- und Seitensträngen auftreten können, folgen den von der Peripherie eintretenden Septen nit ihren Gefäßen. Dadurch kommt, trotz fleckiger, rugleich eine gewisse symmetrische Anordnung zustande. Indem M. damit die Abhängigkeit der Degenerationen von der Gefäßverbreitung hervorhebt, betont er zugleich, daß es sich nicht um eine Schädigung der Nervensubstanz nach Systenten handle. v. Noorden geht in seinen Suhlüssen noch weiter, indem er meint, daß es sich nicht um einen Entzïndungsproze., , sondern um einen akuten Degenerationsprozeß handle.

In dem gleichen Jahre erscbienen noch einschlägige Arbeiten von Eisenlohr, Leyden und Bulloch. Eisenlohr teilte einen Fall mit, der anatomisch insofern etwas Neues bot, als sich bei ihm zahlreiche Blutungen in der grauen Substanz gefunden hatten. Leyden weist die Veränderungen an Hand eires Falles in das Gebiet der cbronischen Myelitis, während Bulloch die Einlagerung hyaliner Massen zwischen die Fasern der weißen Substanz, in die Ganglienzellen der Vorderhörner und der Gefäßwandungen als Ursache der Degenerationen ansieht.

Im Jahre 1893 trat Nonne zum ersten Male mit dem Frgebnis seiner Untersuchungen über unscren Gegenstand, dem er von nun an stets unvermindertes Interesse angedeihen lie $\beta$, in die Öffentlichkeit. Die bekannt gegebenen Fälle bewiesen, daß die perniziöse Anämie durchaus nicht immer das Primäre zu sein brauchte, daß vielmehr 
manifeste Rückenmarlsssvmptome den Erscheinungen der Anämie vorauseilen konnten. Während die Anämie zum Tode führte, konnte die Entwicklung der Spinalsymptome lange vorher zum Stillstand, ja das Spinalleiden zur Rückbildung gelangt sein. Diese Beobachtung sprach ijberzeugend gegen eine pathogenetische Abhängigkeit des Spinalleidens von der Anämie. Sie zeigten weiterhin, was schon durch Eisenlohrs Fall belrannt geworden war, daß die Spinalerkrankung besagter Art durchaus nicht nur mit der perniziösen Form, sondern vielmehr auch mit leichteren letal verlanfenden Arämien einhergehen konnter. Zum Unterschied gegen die Tabes dorsalis hebt N. hervor, daß ätiologisch die Lues keine Rolle spiele, dab es sich im Gegensatz zur Tabes um einen akuten Prozeß handle, der durch abweichende klinische Symptome hinreichend charakterisiert sei und daß die anatomische Lokalisation eine wesentlich andere sei: Die Lissauerschen Zonen seien nie, die Clarkeschen Säulen höchst selten ergriffen; nie findet man eine wesentliche Erkrankung der hinteren Wurzeln, nie eine Schrumpfung der Hinterstränge. Als Hauptort der Frkrankung hat das Halsmark zu gelten, im Gegensatz zu der Tabes, die sich hauptsächlich im Lendenmark lokalisiert. Die von Minnich erhobenen Gefäßbefunde vermag Nonne zu bestätigen. Er findet das Lumen der Gefäße häufig verengt und einzelne sogar verschlossen. Dio Nervensubstanz zeigt Zeichen akuten Zerfalls: Stärlsste Quellung der Markscheiden bei zurücktretender Gliawucherung.

Weitere z. T. umfangreiche Arbeiten knüpfen sich an die Namen Arning, Birulja, Bowman, Rösebeek (1894), denen sich im Jahre 1895 Petrén und Burr anschließen, ohne wesentlich neue Gesichtspunkte unserer Frage beizubringen. Taylor (1894) indessen will die in seinem Falle beobachteten Blutungen als Ursache für die Entstehung der Degenerationsherde heranziehen, eine Frklärung, die schon vor ihm von einigen Autoren in Erwähnung gezoger, aber abgelehnt worden ist.

Einen neven sehr bestechenden Gedanken brachte Rothmann (1895) in die Diskussion. Er suchte die primäre Erkrankung im Bereiche der grauen Substanz, wo er eine deutliche Rarefikation der Vorderhörner mit Blutungen gefunden hatte. Diese sollten die Uriache für eine sekundäre Degeneration der Bahnen in der weißen Substanz sein. Fr erblickte in den in Rede stehenden Krankheitsfällen kombinierte Systemerkankungen im Sinne von Kahler und Pick. Bastianelli äußerte sich in gleicher Weise. 
In den Jahren 1895/96 teilte Nonne die Untersuchungsergebnisse von insgesamt 19 neuen Fällen mit. Darunter befanden sich drei Fälle frischester Entstebung. Sie zeigten, daß das Leiden mit der Bildung einzeln aufschließender, schlief,ich konfluierender Herde im Halsmark zu beginnen pflegte. Dabei betonte Nonne die nach seiner Meinung sicher bestehende Abhängigkeit der Degenerationen von den GefäLen und wandte sich entschieden gegen die Pothmannsche Auffassung, mit der Begründung, daß die Vorderhornveränderungen im Vergleich mit der Ausdehnung der Strangdegenerationen viol zu geringfügig seien, um ursächlich beteiligt sein zu können. Dieser Ansicht traten die meisten späterer Autoren bej.

Während I'eichmüller im Jahre 1896 aư Grund seiner Untersuchungen der Auffassung Taylors beitrat, daß die Degererationen Folge zahlreicher Blutungen in die weiße Rückenmarkssubstanz sein sollte, brachten die von Lloyd, Matthes, Müller, Lenoble und Clarke veröffentlichten Fälle im wesentlichen die Bestätigung der von Minnich, Nonne u. a, erhobenen Befunde. v. Voß (1897) hoffte die Frage des Zusammenhanges von Anämie und Rückenmarksveränderungen auf dem Wege des Fxperimentes klären zu können. Es gelang ihm, durch häufige Entziehung geringer Blutmengen oder durch Injektion blutschrädigender Stoffe (Glyzerin, Pyrogallol) bei Tieren hochgradige Anämie zu erzeugen und diese in diesem Zustande längere Zeit am Leben zu erhalten. Das negative Ergebnis der nach dem Tonde vorgenommenen Rückenmarksuntersuchung bewies nur, daß Blutverlust allein nicht imstande ist, Rückenmarksteränderungen herbeizuführen. An dieser auf einwandfreie Weise gewonnenen Tatsache vermochte auch der später vor Ransoboff (1899) mitgeteilte Fall nicht zu rütteln.

Neben den Veröffentlichungen von Brasch, Goebel, Johnson, StrauB ist für das Jahr 1898 hauptsächlich der umfangreichen Arbeit von Boedecker und Juliusburger zu gedenken, die sich in ihren Endergebnissen Nonne anschließt. Die Verfasser heben aber als erste besonders hervor, dafi die geschilderten Rückenmarksveränderungen keineswegs nur für perniziöse Anämie charakteristisch wären. Tielmehr habe schon Lichtheim (1890) ähnliche Veränderungen bei kachektisierenden Krankheiten verschiedener $\dot{A}$ tiologie, sie selbst bei Karzinose beobachtet.

Das Jahr 1899 brachte wieder eine umfangreiche Arbeit von 
Nonne. Neben einer Znsammenstellung aller bisher bekannt gewordenen Untersuchungsergebnisse teilte sie 6 nene Fälle mit und verbreitete sich anschließend über die von Nonne angenommene Pathogenese des Prozesses. „Die Degenerationen sind zum gröBten Teil rnn unregelmäßiger Verbreiturg, reder stets symmetrischer Art, noch an bestimmte Neurongruppen gebunden." Dieser Arsicht Schu?tzes cchließt sich Nonne an und fügt erklärend hinzu: ,Es ist klar, daß, wenn an umschriebene Degenerationen, speziell an durch Konfluenz kleinerer Herdo entstandene, ausgedehntere Degenerationen, eine solche in den zugehörigen Babnen sich anschließt, der Suheis einer primären Strargerkrankung erweckt werden kann": Diese ist also sekundär bedingt, akhängig von den Gefäßen. Das hypothetische Toxin wird durch die Gefäße transportiort, schädigt anfangs richt diese, sondern die Nervensubstanz. Frst bei längerer Dauer der Einwirkung Ieiden auch die Gefäßwände und verursachen dadurch eine Ernährungsstörung, die die Toxine in ilırer Wirkung unterstützen. Den Beweis für die Richtiglieit der Gefäßtheorie sucht Nonne durch die Untcrsuching von Rückenmarken in Fällen ron Sepsis zu erbringen. Hier, wo ja die Wirkung eines Toxins auf dem Wege der Gefäße als Tatsache anzusehen ist, kounte Nonne die gleichen Veränderunger feststellen, wio sie von ihm bej pcrniziöser Anämie in den sog. Frühfällen erhoben worden waren: „Was hier im Ausch!̣ß an die Gefäße langsam und allmählich zustande kommt, das sehen wir in akuter Weise bei den Anämien auftreten! Wenn Nonne den Prozeß als Myelitis bezeichnet, obgleich eigentliche entziindliche Infiltrate der Blutgefäßwandungen meist fehlen, so ist er sich klar darïiber, daß es sich nicht um sigentliche Entziindung handelt, sondern hauptsächlich um eine subakute Erweichung, die durch eine die Zirkulation in den Gefäßen störende Schädlichkeit hervorgerufen wird. Unter Hinweis auf eine Arbeit Russels hebt Nonne hervor, daß vielfach Rückenmarkserkrankungen in den Bereich der Betrachtung gezogen und mit den Spinalveränderungen bei perniziöser Anämie in eine Reibe gestellt wurden, die primàr als kombinierte Systemerkrankungen in Sinne von Kahler und Pick und Strümpell mit später terminal komplizierender Anämie aufzufassen seien. Hier möchte aber streng unte ${ }_{\lrcorner}$schieden werden. Jene Fälle echter kombivierter Systemerkrankung lassen die Entstehung der Degenerationen aus einzelnen Herden nicht erkennen.

Trotz der Beobachtung schwerer Gefäßveränderungen - adventitielle Infiltration und ausgedehnte Intimawucherung - vermögen 
Jakob und Moxter (1899) diesen in der Pathogenese dos Prozesses nisht eine bedeutsame Rolle zuzuerkonnen.

Während Marburg (1900) sich weder für die myelitische noch für die myelomalacische Genese zu entscheiden vermag, tritt Rheinboldt (1901) für die entzündliche ein. Er nimmt an, „daß das Gift einen mehr akuten Faserzerfall in der unmittelbaren Nachbarschaft der Gefäße, bєi seiner durch die Kapillaren erfolgenden Ausbreitung einen weniger akut verlaufenden Faserschwund verursaiken". Dadurch kommen, wenn man will, zwei verschiedene Formen zustande: Die perniziös anämischen Spuinalerkrankungen und die kombinierten Systemerkrankungen mit yerniziöser Anämic. Beide Formen möchte Rh. in eine große Gruppe anämiseher Spinalerkrankungen unter demi einheitlichen Gesichtspunkte der vaskulär-toxischen Pathogenese zusammengefaßt wissen. $\mathrm{Zu}$ beiden Formen steht die Anämie verschiedensten Grades nicht in direlkt ätiologischer Beziehung; doch sind indirelste Beziehungen zu beiden Formen sicher anzunehmen. „Fine Scheidung der als anämische Spinalerkrankung mit vaskulärer Pathogenese zusammengefaßte Grupne ist in dem Sinne für die Praxis aufrecht zu erhalten, als man sagen kann, daB es bei letaler Anämie vorwiegend zu herdförmigen Erkrankungen, bei nicht letalen leichteren Anämien vorwiegend zu diffuser Sklerose mit strangförmiger Ausbreitung kommt". Mit dieser Ansicht trat $\mathrm{Rh}$. in bewußten Gegensatz zu Nonnes dualistischer Auffassung. Tinem Vorschlage Hennebergs folgend führt dieser für seine herdförmig-konfluierenden Rückenmarksdegenerationen die Bezeichnung: "Pseudosystemerlzrankung" ein. In Arbeiten der Jahre 1906/07 kommt, er zu dem Ergebnis, daß die Spinalveränderungen keineswegs an das Bestehen einer Anämie gebunden seien.

Nachdem in den folgenden Jahren durch Batten, Church, Dinkler, Markus u. a. weiteres Material bekannt geworden war, erschien im Jahre 1908 eine größere Arbeit von Nonne und Fründ. Dain nähern sich die Autoren der Ansicht Rheinboldts, indem sie den strengen Unterschied zwischen den beiden Formen der Spinalerkrankungen, den erhten lombiniert systematischen und den pseudosystematischen fallen lassen. Sie weisen nach, daß an dem Vorkommen echter kombinierter Systemerkrankungen mit Ausnahm e der Tabes, der Friedreichschen Ataxi e und der amyotrophischen Lateralsklerose mit Recht zu zweifeln sei und kommen zu der Überzeugung, daß auch die von Nonne bisher als kombinierte Systemerkrankungen abge- 
sonderten Fälle sowie dic ,klassischen Fälle von echter Systemerkrankung" der Iiteratur aus einzelnen Herden in der Umgebung der Gefäße entstanden seien. Die gesonderte Versorgung der einzelnen Stranggebiete durch verschiedene Gefäße läßt das Zustandekommen strangförmiger Degenerationen erklärlich erscheinen. Für die systematische Degeneration der Kl. H. S. Str. macht Nonne außerdem die Unterbrechung der von den Clarkeschen Säulen kommenden Fasern im Bereiche der erkrankten Pyramidenstränge verantwortlich. - Für die Pathogenese der Veränderungen in der Umgebung der Gefäße muß man unterscheiden zwischen den Tällen akuter Entstehung mit kleinzelliger Infiltration der Gefäße, als Zeichen einer toxämischen Erkrankung und den älteren Fällen mit hyalin entarteten Gefäßwandungen, die das Zeichen für ischämische Entstehung der Degenerațon abgeben. Beide Prozesse können sich vereinigen.

In den folgenden Jahren crschienen Arbeiten von Siemerling (1909), Henneberg (1911), Richter (1912) u. a., die in den Grundzügen den Nonneschen Ansichten folgten. Das Jahr 1913 brachte Arbeiten von Dinkler, Knoch, Lube und Lenel, das Jahr 1916 eine kurze Mitteilung von Rößle zu unserem Gegenstande. Dinkler kommt zu dem Schluß, daß die Spinalveränderungen sicherlich nicht durch das gleiche Toxin bewirkt werden, wie die Blutveränderungen, denn dafür seien jene bei Anämie zu selten. Lube vermag in seinen Fällen die Abhängigkeit der Degenerationen von den Gefäßen nicht anzuerkennen, ohne damit bezweifeln zu wollen, daß diese in Minnichs und Nonnes Fällen bestanden haben. Aber es erscheint ihm möglich, daß in einem Teil der Fälle die Ausbreitung der Degenerationen unabhängig von der Gefäßerkrankung, lediglich durch eine besondere Affinität des Toxins zur weißen Substanz erfolgen könne. Lenel hingegen vermag sich keiner der bisher bekannt gewordenen Theorien für die Pathogenese anzuschließen und läßt die Frage als ungelöst offen.

Wie das Studium der Literatur gezeigt hat, ist die Auffassung der durch Lichtheim bekannt gewordenen Spinalerkrankungen im Laufe der Jahre wesentlichen Wandlungen unterworfen gewesen. Die Klärung der mannigfaltigen interessierenden Fragen war besonders dadurch erschwert, daß diese auf der einen Seite eng mit der noch jetzt teilweise unbekannten Ätiologie und Pathogenese der schweren und perniziösen Anämien verknüpft erschien, auf der anderen Seite stark durch die auseinandergehende Auffassung wichtiger neuropatho- 
logischer Begriffe beeinflußt wurde. So finden wir die Tehre von den kombinierten Systemerlzrankungen und der Myelitis eng verkniupft mit der Forschung über die hier in Pede stehenden Rückenmarkserkrankungen. Wenn auch heute noch bei weitem nicht in allen Punkten Übereinstimmung erzielt worden ist, so kann man doch in der von Nonne und Fründ (1908) erschienenen Arbeit einen gewissen Abschlnß erblicken. Eine zusammenfassende Schilderung ron dem heutigen Stande der Frage schließt sich deshalb eng an diese Arbeit an, um so mehr, als die ihr in den vergangenen Jahren folgenden Veröffentlichungen. sich in den Hauptpunkten mit Nonnes Ansichten decken. In diesem Sinne sei auf Hennebergs Ausführungen über ,funikuläre Myelitis" in Lewandowskys ,Handbuch der Neurologie“" verwiesen.

Mit wenigen Worten finden wir die Rückenmarkserkrankung in Lewandowskys, ,Praktische Neurologie für Ärzte“ 1919 charakterisiert: ,Die praktisch wichtigsten der diffusen Rückenmarkserkrankungen sind die bei schweren Pluterkrankungén und bei Kachexie auftretenden. Es sind das zu einem großen Teil diejenigen Fälle, welche man früher als kombinierte Strangerkrankungen bezeichnete, bis es sich heralusstellte, daß es sich bei diesen Erkrankungen um nichtsystematische außerordentlich ausgedehnte, leichte myelitische Veränderungen handelte (,funikuläre Myelitis" ${ }^{6}$ ). Diese Erkrankungen kommen vor bei perniziöser Anämie, bei Leulämie, bei Karzinomkachexie und anderen kachektischen Zuständen, am ausgedehntesten bei den erstgenannten Blutkrankheiten".

Die Mitteilung zweier einschlägiger Fälle aus dem Material des Herm Geheimrat Schmorl, dem ich, ebenso wie Herrn Prof. Päßler, für die Überlassung des Materials zum Danke verpflichtet bin, soll dazu dienen, die noch der Klärung harrenden Fragen der Lösung näher zu bringen.

Fall 1. Krankengeschichte (gekürzt, aus dem Res.-Laz. I Dresden).

Offizierstellvertreter K., aktiver Soldat, 37 Jahre.

Vorgeschichte: Patient ist mit gesunder Frau verheiratet, Vater eines gesunden Kindes, während ein Kind mit 4 Monaten an allgerneiner Schwäche starb. Keine Fehlgeburt. Sonst Familienanamnese o. B.

Patient selbst will nie ernstlich krank gewesen sein, insbesondere nie geschlechtskrank. Kein Alkoholmißbrauch. Während des Feldzuges in China, den Patient während 11/4 Jahren (1900-1901) mitmachte, will Patient 6 Tage sehr starker Kälte ausgesetzt und danach krank gewesen sein. Im Jahre 1906 trat plötzlich zum ersten Male allgemeine Mattigkeit, 
Schwäche in den Beinen und Schwindel auf. Diese Anfälle wiederholten sich jährlich zwei- bis dreimal. Seit 1915 nahmen die Schwächeanfälle zu. Atemnot trat auf. Krankmeldung am 15. IV. 1916.

Befund am 15. V. 1916 (durch Truppenarzt zum Zweck eines Gutachtens erhoben).

K. klagt über allgemeine Schwäche, Schwindel, Unsicherheit beim Gehen, Steifigkeit in den Unterschenkeln, Atemnot, sowie Kälte und Gürtelgefühl.

Körpergröße: 1,58 m, Körpergewicht 49,5 kg. Starke Abmagerung, schlaffe Muskulatur. Haut gelblich, die Bindehaut ikterisch.

Herz: Ohne nachweisbaren krankhaften Befund.

Lungen: Über der rechten Lunge hinten zeitweise Rasselgeräusche, sonst kein krankhafter Befund.

Leber und Milz: Nicht vergrößert.

Nervensystem: Pupillen gleichweit, bei etwas träger Reaktion. Gaumenreflex nicht auslösbar, Kornealreflex herabgesetzt, Bauchdeckenreflexe +, Patellar- und Achillessehnenreflexe fehlen beiderseits. Romberg + , ataktischer Gang.

Wassermannsche Reaktion im Blut negativ. - Urin frei von Eiweiß und Zucker.

Krankheitsbezeichnung: Tabes dorsalis.

28. V. Lazarettaufnahme.

Befund: Patient ist ziemlich stark benommen. Am Herzen ist eine Verbreiterung nach links festzustellen, außerdem ein weiches systolisches Geräusch an der Pulmonalis.

Puls untermittelkräftig, regelmäßig beschleunigt. Druck $116 \mathrm{~m} / \mathrm{Hg}$.

Lungen ohne Besonderheiten.

Leber vergrößert, unterer Leberrand in dex Brustwarzenlinie einen Querfinger unter dem Rippenbogen. Oberfläche und Rand glatt.

Ödem an beiden Füßen und Knöcheln.

Nervensystem: Pupillen links $=$ rechts, reagieren vielleicht etwas träge. Kein Nystagmus. Kein Einstellungszittern. Bindehautreflexe erhalten. Würgereflex aufgehoben. Bauchdeckenreflex beiderseits auslösbar.

Kremestarreflex rechts $=$ links.

Patellarreflex rechts fehlend, links schwach positiv.

Achillessehnenreflexe beiderseits fehlend.

Kein Babinski.

Knie-Hackenversuch uncharakteristisch.

Keine sensiblen Störungen.

Stark ausge prägter Romberg.

Der Gang fällt auf durch leicht lordotische Haltung der Wirbelsäule mit leicht zurückgebogenem Kopfe. Der Gang ist weder ataktisch, noch spastisch, mehr kurzschrittig.

Wassermannsche Reaktion im Blut negativ.

Temperatur: 37,8 . 
Augenhintergrund: mehrere kleine retinale Blutungen.

31. V. Geringe abendliche Temperaturerhöhungen, Sensorium freier. Stuhlgang nicht acholisch. Im Marn kein Gallenfarbstoff nach Gmelin und Rosin nachweisbar. Kein Aszites.

15. VI. Nach vorübergehender Besserung des Allgemeinbefindens, bei unverändertem Organbefund, ist jetzt wesentliche Verschlechtexung eingetreten. Starke Benommenheit. Ödeme an den Unterschenkeln bis zum Knie, später bis zur Hüfte. Beugekontrakturstellung des linken Vorderarmes. Häufige diarrhöische Stuhlentleerung.

28. VT. Sehr unruhig, zeitlich und örtlich sehr ungenau orientiert; konfabuliert etwas. Neurologischer Befund im allgemeinen unverändert. Bauchdeckenreflexe beiderseits gut auslösbar.

5. VII. Verfall; benommen, gähnt viel. In der Nacht ziemlich unruhig, schreit laut. Exitus letalis.

Sektionsbefund (Geh. Med.-Rat Prof. Dr. Schmorl): Allgemeine Blutarmut. Zahlreiche punktförmige Blutungen im Gehirn, vereinzelte auch in den unteren Abschnitten des Rückenmarks. Graue Degeneration in der rechten Kleinhirnseitenstrangbahn.

Hochgradige wäßrige Durchtränkung der Lungen. Fettige Entartung des Herzfleisches; Eirweiterung der Herzkammern. Brust- und Bauchwassersucht. Milzschwellung. Nierenentzündung. Eisenablagerung in der Leber. Rotes Mark im Schafte des Oberschenkelknochens. Blutungen auf dem Herzbeutel, dem Lungenfell sowie auf der Netzhaut des Auges. 歌. Nach Härtung in Müller-Formalin gibt die Betrachtung der Rückenmarksquerschnitte folgenden Befund:

Halsmark: dichte, weiße, scharf abgesetzte, symmetrische Aufhellung in den Hintersträngen (H.S.), die die dorsale Hälfte der Burdachschen Stränge einnimmt, während die Gollschen Stränge unverändert erscheinen. Die Kleinhirnseitenstränge (Kl.H.S.S.) sind scharf gegen die Umgebung auf beiden Seiten symmetrisch als weißes Degenerationsfeld abgegrenzt, während die Pyramidenseitenstränge (Py.S.) nur einzelne helle kleinste Flecken erkennen lassen und die Gegend der Vorderstränge unverändert ist.

Brustmark: Die Herde in den Hintor- und Seitensträngen sind fleckiger und weniger scharf gegen die normal erscheinende Umgebung abgesetzt. In den kaudalen Teilen des Brustmarks reicht die Aufhellung in fleckiger Anordnung bis in die ventralen Teile des Keilstranges, läßt aber das ventrale Hinterstrangfeld und einen schmalen Saum neben den Hinterbörnern frei. In den Seitensträngen hat sich die Aufhellung im Bereich dєr Py.S. ausgebreitet, hält dabei aber völlige Symmetrie und den fleckigen Charakter bei. Die Vorderstränge sind nicht erkennbar verändert.

Lendenmark: zeigt keine Abweichungen von der Norm.

Mikroskopischer Befund: Der mikroskopischen Untersuchung wurde das Rückenmark in 12 verschiedenen Höhen unterzogen unter Vornahme folgender Färbemethoden: Markscheidenfärbung nach Weigert, 
mit Sudan III, Gliafärbung nach van Gieson, mit Hämatoxylin-Eosin, Ganglienzellenfärbung mit Thionin.

Oberes Halsmark: Das dorsale Drittel der Keilstränge zeigt beiderseits unregelmäßigen fleckigen Faserausfall, der an der Peripherie des Marks am dichtesten ist und dort keinerlei Markscheiden und Achsenzylinderstruktur mehr erkennen läßt. In ventraler Richtung verlieren sich die Degenerationen mehr und mehr in gesundes Gebiet, dort findet man neben vorwiegend unverändert gebliebenen Nervenfasern, vereinzelt solche, deren Achsenzylinder gequollen, deren Markscheiden gebläht sind. Medial vom Septum intermedium, unmittelbar an dieses angeschlossen, liegen auch im Gollschen Strange Degenerationsherde, die ihre größte Ausdehnung ebenfalls an der Peripherie des Marks besitzen, im ganzen aber unbedeutend sind. Die Wurzeleintrittszone und Lissauersche Randzone sind intakt. An Stelle der zugrunde gegangenen Nervensubstanz findet sich ein System von Gliamaschen, die teilweise leer, teilweise mit durch Sudan leuchtend gefärbten Zerfallprodukten in mäßiger Menge erfüllt sind. Die Glia ist nirgends gewuchert.

Die Kl.H.S.Str. sind vollkommen zerstört. Man findet in ihrem Gebiet, das sich ziemlich scharf gegen die benachbarten mehr oder weniger unveränderten Stränge abgrenzt, keine Markscheiden mehr. An ibrer Stelle ist jenes oben beschriebene, besonders weitmaschige Gliafasergerüst. In den Py.S.Str. findet sich vereinzelter Faserausfall neben gequollenen Achsenzylindern und Markscheiden. In den Vordersträngen ist nur ganz vereinzelter Faserausfall, ohne erkennbare geregelte Anordnung nachzuweisen.

Die Wandungen der Gefäße zeigen nur in den Degenerationsbezirken, und auch dort nicht durchgehend, geringe kleinzellige Infiltration. Wandverdickung durch Quellung oder durch Einlagerung hyaliner Massen wird nirgends beobachtet. Keine Blutaustritte.

Die graue Substanz läßt bis auf geringe Fettoinlagerung in die Ganglienzellen der Vorderhörner keine Veränderungen erkennen.

Periphere Wurzeln und Pia mater sind intakt.

Unteres Halsmark: Die Hinterstränge zeigen dasselbe Bild wie oben. In den Seitensträngen schieben sich die Degenerationen mehr in die Pyramidenstränge hinein, während die Entartung der Kl.H.S.Str. rechts weniger scharf gegen die Nachbarbahnen abgegrenzt ist. In dem rachten Py.Str. findet sich ein kleinster Herd.

Oberes Brustmark: Die Degenerationsbezirke in den Hintersträngen sind anfangs nicht verändert. Sie erstrecken sich aber, je weiter man nach unten gelangt, mehr in die ventralen Teile, indem dabei der herdförmige Charakter der Entartung vollkommen gewahrt bleibt. Der Degenerationsbezirk an der Basis nimmt an Ausdehnung ab. Im ventralen Teile des linken Keilstranges findet sich ein kleiner Herd frischer Entstehung, in dem die erhaltenen, doch gequollenen Markscheiden im Sudanpräparat durch leuchtende Rotfärbung ihren beginnenden Zerfall anzeigen. In den Seitensträngen weichen die Degenerationen zentralwärts und breiten 
sich regellos, ganz unsystematisch über das Gebiet aus. Rechts ist der Faserzerfall stärker in den Pyramiden als in den Kl.H.S.Str.; links verhält es sich umgehehrt. In den Py.S.Str. nur vereinzelter Faserausfall.

Unteres Brustmark: Die Hinter- und Seitenstrangdegenerationsbezirke bleiben sich im allgemeinen gleich. Ihre Ausdehnung ändert sich nur ganz unbedeutend und ganz unregelmäßig. Die Vorderstränge sind fast ganz unverändert.

Lendenmark: In den unteren Teilen ist die Nervensubstanz unverändert, während die oberen Abschnitte denen des unteren Brustmarkes gleichen. Die Gefäße zeigen keinerlei Veränderungen gegenüber der Norm, während sie in den oberen Markschnitten die schon oben beschriebene geringe Kernwucherung in der Adventitia erkennen lassen. Dabei handelt es sich um Lymphocyten. In den perivaskulären Lymphknoten sind Körnchenzellen nur ganz vereinzelt auffindbar.

Von den Brust- und Bauchorganen zeigt das Herz fettige Degeneration, das Fett ist in die Muskelfasern, zu beiden Seiten der Kerne eingelegt.

In der Leber finden sich neben ausgesprochen positiver Berlinerblaureaktion kleinste Nekroseherde (das Pigment liegt in den Leberzellen). Das Protoplasma der Leberzellen ist darin getrübt, die Kerne verschwunden. Auch in den Nieren reichliche Ablagerung von eisenhaltigem Pigment. Die Glomerulusschlingen sind geschwollen, sehr zellreich und füllen die Kapsel prall an.

Fall 2. Krankengeschichte (aus der I. Inneren Abteilung des Friedrichstädter Krankenhauses, Prof. Dr. Päßler).

M., Gutsbesitzersehefrau, 49 Jahre.

Vorgeschichte: Familienanamnese ohne Besonderheiten. Patientin hat drai gesunde Kinder. Pat. hat früher mehrmals Pleuritis gehabt, die vom behandelnden Arzt als tuberkulös betrachtet wurde.

Seit Weihnachten 1918 hat Pat. Schmerzen in den Beinen und taubes Gefühl in den Fußsohlen. Allmählich stellte sich Schwäche in den Beinen ein, die stsif wurden und das Gehen unmöglich machten. Seit September 1919 sind die Schmerzen in den Beinen behoben, die Füße sind wie Klötze. Wasserlassen ohne Beschwerden. Gebrauch der Arme gut möglich. Appetit sehlecht. $\mathrm{Ab}$ und zu Erbrechen und Magenschmerzen. Zunehmende Blässe des Gesichts. Klagen über Schmerzen in der Zunge mit häufiger Bläschenbildung darauf. Krankenhausaufnahme am 24. IX. 1919.

Befund: Ziemlich anämisch aussehende Frau in schlechtem Ernährungszustand. Zunge oberflächlich atrophisch.

An den Brust- und Unterleibsorganen ist ein krankhafter Befund nicht zu erheben.

Puls regelmäßig, mäßig gefüllt und gespannt.

Blutdruck $110 \mathrm{~mm}$ Hg nach Riva-Rocei.

Blutbefund $40 \% \mathrm{Hb}$ (Antenrieth); 2220000 Erythrocyten, 1500 Ləukocyten im Kubikmillimeter. Färbeindex 0,9; Poikilo- und AnisoCytose.

Urin frei von Eiweiß und Zucker. 
Nervensystem: ausgesprochener horizontaler Nystagmus beim Blick nach rechts wie nach links. Sonst Hirnnerven intakt.

Bauchdeckenreflexe fehlen beiderseits.

Patellar- und Achillessehnenreflexe sehr lebhaft, l. = r. beiderseits Patellar- und Fußklonus.

Babinski beiderseits positiv.

Mastdarm und Blase intakt.

Sprache gut, etwas langsam.

Grobe Kraft in den Armen gut.

Die Beine können nur wenig angezogen werden.

Kein Intentionszittern.

Hypertonie der Muskulatur der unteren Extremitäten.

Sensibilität: Klagen über Parästhesien in den Beinen. Lageempfindung in den Zehengelenken, Fuß- und Kniegelenke auffallend gestört. Berührungsempfindung an den U.-Extremitäten beiderseits aufgehoben, und am Rumpf bis zu den Brustwarzen abgeschwächt. Schmerzempfindung am rechten Bein fast aufgehoben, am linken herabgesetzt. Wärmeund Kälteempfindung am rechten Bein stärker, am linken Beine weniger stark gestört.

Salzsäurereaktion im erbrochenen Mageninhalt negativ.

Lumbalpunktion: Flüssigkeit klar, Druck $140 \mathrm{~mm}$ Wasser, starke Atemschwankungen, Nonne negativ. Fuchs-Rosenthal: im ganzen 3 Zellen, verschiedene Erythrocyten. Wassermann im Liquor negativ.

1. XI. 1919. Verlauf: Pat. klagt über spannendes Gefühl in den Beinen, wo zeitweise Schmerzen auftreten. Schwäche in den Armen, taubes Gefühl in den Fingern. An der Zunge schmerzhafte rote Stellen.

6. XI. Hb. $45 \%$ Erythrocyten 2580000 , Anisocytose, geringe Polikilocytose. Leukocyten 3000.

28. XI. Zeitweise heftige krampfartige Schmerzen in den Beinen. Keine Entartungsreaktion. Zunehmende körperliche Schwäche, läßt StuhI und Urin unter sich. Sehnenreflexe an den unteren Extremitäten schwächer. Kein Klonus. Keine Babinski mehr.

1. XII. Auf intravenöse Blutinjektion vor 3 Tagen heute $\mathrm{Hb} .53 \%$, Erythrocyten 2500000 . Schmerzen in den Beinen lassen nach.

9. XII. Hb. $40 \%$. Erythrocyten 2000000 . Starke Muskelatrophie an den Beinen. Sehnenreflexe aufgehoben.

13. XII. Bei zunehmendem Verfall erfolgt unter den Erscheinungen einer doppelseitigen Pneumonie der Tod.

Sektionsbefund (Geh. Med.-Rat Prof. Dr. Schmorl):

Chronische Lungentuberkulose. Ausgedehnte verheilende Hohlgeschwüre im linken Oberlappen, zahlreiche Aspirationsherde im linken Unterlappen. Frische Pneumonien im rechten Unterlappen. Daselbst vereinzelte tuberkulöse Herde, zahlreichere im Mittellappen. Tuberkulose der Bronchiallymphknoten. Pleuritis exsudativa. Bronchitis. Tracheitis. Tuberkulöse Geschwüre im Dickdarm, an der Valvula Bauhini und im unteren Dünndarm. Struma colloides. Anämie und Verfettung des Herz- 
fleisches. Stauung in den Unterleibsorganen. Milztumor. Allgemeine Anämie und Kachexie. Atrophie der Genitalien. Schwere Cystitis. Thromben im Plexus pubicus. Chronische Metritis. Gallensteine.

Nach Härtung in Müller-Formalin ergibt die Betrachtung der Rückenmarksquerschnitte folgenden Befund:

Halsmark: Dichte weiße Aufhellung im Bereiche der Hinterstränge, unter Freilassung eines schmalen Saumes entlang der granen Substanz und - im oberen Halsmark - auch des ventralen Hinterstrangfeldes. Im Boreiche der Burdachschen Stränge erscheint die Degeneration fleckiger als in den Gollschen Strängen. Dichte weiße Aufhellung an den Seitensträngen. Im oberen Halsmark sind die Kleinhirnseitenstränge (Kl.H.S.Str.) am stärksten ergriffen und zeichnen sich von der fleckigen Degentration der kortikofugalen Bahnen durch ihre gleichmäBige Aufhellung aus. In dem unteren Halsmark sind auch diese gleichmäßig grauweiß gefärbt. Der Pyramidenvorderstrang zeigt nur im mittleren Halsmark beiderseits feinste Fleckung. Das Bild ist überall so gut wie symmetrisch.

Brustmark: Die Hinterstränge zeigen in allen Teilen eine fast vollkommen gleichmäßige grauweiße Verfärbung. Das ventrale Hinterstrangfeld ist stellenweise, aber durchaus nicht in allen Höhen, freigeblieben, im Gegensatz zu den den Hinterhörnern innen anliegenden Partien. Im untersten Teil wird das Degenerationsfeld kleiner. Es läßt jetzt deutlich auch die dem Septum posterius anliegenden Teile frei. Die Seitenstränge sind oben wie unten gleichmäBig grauweiß gefärbt. Nach den Vordersträngen zu zeigt sich besonders in den unteren Teilen entlang der Peripherie fleckige Aufhellung, die sich im Berejche $d \in r$ Py.S.Str. nur im mittleren Brustmark stärker ausprägt.

Lendenmark: Die Degenerationsfelder werden kaudalwärts immer kleiner und lassen in den Hintersträngen nach dem Sept. post., den Hinterhörnern und der Peripherie za breitere Felder unberührt. Im tiefsten Teile finden sich nur einige helle Flecken, ebenso verhält es sich mit den Seitensträngen. Die Vorderstränge єrscheinen unverändert.

Mikroskopischer Befund: D $\in$ r mikroskopischen Untersuchung wurde das Rückenmark in 15 verschiedenen Höhen unterzogen unter Vornahme folgender Färbemethoden: Markscheidenfärbung nach Spielmeyer, Fettfärbung mit Sudan III, Gliafärbung nach van Gieson, HämatoxylinEosinfärbung, Elastikafärbung. Gangliẹnzellenfärbung mit Thionin.

Obexes Halsmark: Vollkommene Verödung der Gollschen Stränge, in deren Bereich nur ganz vereinzelte Achsenzylinder und Markscheiden erhalten sind, und zwar hauptsächlich im dorsalen und dem am weitesten ventral gelegenen Abschnitte. Die Burdachschen Stränge zeigen ausgedehnte fleckig herdförmige Degeneration mit reichlich exhaltener Nervensubstanz. Doch ist auch dort an der Basis die Verödung eine totale. Da wo Markscheiden erhalten sind, erscheinen sie meist gebläht, die Achsenzylinder gequollen. Frei von Veränderungen blieb in den Hintersträngen das ventrale Feld, die der grauen Substanz unmittelbar anliegenden Teile und die Wurzeleintrittszone. An Stelle der zerfallenen Nervensubstanz 
finden sich maschenartige Räume von Gliafasern gebildet. Diese sind teilweise mit homogen oder bröcklig erscheinenden Zerfallsmassen angefüllt, die sich mit Sudan rot färben und sich als Lipoide zu erkennen geben oder sie sind vollkommen leer. Die Glia ist nirgends vermehrt, ebenso nicht ihre Kerne. Die Gefäße sind wenig gefüllt und zeigen im Gebiete der Degenerationen dichte Infiltration mit Zellen vom Charakter der Lymphocyten und der Plasmazellen. Verdickung und hyaline Entartung der Wandungen ist nicht festzustellen. Die elastischen Membranen sind intakt. Das Lumen nirgends verschlossen oder eingeengt. In den erweiterten perivaskulären Lymphscheiden liegen massenhaft Fettkörnchenzellen. Blutaustritte sind nirgends vorhanden.

Von den Seitensträngen sind die Kleinkirnseitenstränge ziemlich gleichmäßig degeneriert, links noch etwas ausgeprägter als rechts. Die Py.S. zeigen fettigen Zerfall mit reichlicher Bildung von Maschen. Die Py.Vo.Str. lassen keine Veränderungen erkennen.

Die Vorderhornganglienzellen zeigen im Sudanpräparate dichte Fetteinlagerung, die den Kern meist vollkommen verdeckt. Doch ist dieser sonst zentral gelegen, die Form der Ganglienzellen nicht verändert, nicht geschrumpft. Da die spezifische Ganglienzellenfärbung mangels geeignete Materials keine guten Ergebnisse lieferte, kann über die Beschaffenheit des Tigroids nichts Genaues ausgesagt werden.

Die Pia mit ihren Gefäßen, sowie die peripheren Wurzeln sind unverändert.

Mittleres Halsmark: Das Querschnittsbild bietet im großen und ganzen nur wenig Änderung dar. Die Degenerationen im Burdachschen Strange reichen mit einzelnen Herden bis in die Wurzeleintrittszone hinein. Der Zerfall in den Py.S.Str. ist einheitlicher. In den Py.S.Str. sind einige Zerfallsherde fleckigen Charakters aufgetreten, die im Fettpräparate ihre frische Entstehung durch Einlagerung leuchtend rot gefärbter Zerfallsprodukte erkennen lassen.

Unteres Halsmark: Die Degeneration der Gollschen Stränge ist total und deutlich in Flaschenform ausgebildet. Im ventralen Felde nur noch ganz vereinzelte Markscheiden erhalten. In den Keilsträngen hingegen ist die Degeneration ausgeprägt herdförmig. Vereinzelter Faserausfall findet sich auch hier in den Wurzeleintrittszonen, die aber im ganzen unverändert bleiben. Seitenstränge wie oben. Py.Vo.Str. enthalten reichlich Zerfallsherde, besonders rechts. Vorderhornganglienzellen und Gefäße wie oben geschildert. Diese sind übrigens in den unveränderten Gebieten des Rückenmarks normal.

Oberes Brustmark: Die Degenerationen in den Gollschen Strängen machen nicht mehr den streng systematisohen Eindruck wie im unteren Halsmark. Sie enthalten reichliche gesund erscheinende Markscheiden, besonders beiderseits vom Septum posterius mediale. Die Burdachschen Stränge sind in ihrer ventralen Hälfte fast vollkommen intakt. Nur das Gebiet der ",mittleren Wurzelzone " ist schwerer verändert. Während die laterale Wurzelzone hier wie überall keine Veränderungen erkennen läßt, 
zeigen sich wieder vereinzelte degenerierte Fasern in der Wurzeleintrittszone.

Die Intensität der Degenerationen in den Kl.H.S.Str. ist geringer geworden, hingegen bedeutend dichter in den Py.Vo.Str. Sie reichen links weiter nach ventral als rechts.

In den Vordersträngen findet sich reichlicher Faserausfall, besonders dicht an der Peripherie des Marks. Er ist älterer Entstehung; denn es lassen sich im Gegensatz zu den anderen Stellen dort keine Lipoide mehr nachweisen.

Mittleres Brustmark: Die Degeneration in den Gollschen Strängen rückt mehr und mehr vom Mittelseptum ab, wo die Zahl der erhaltenen Markscheiden zunimmt. Dafür sind die Zerfallserscheinungen in den Burdachschen Strängen hier entschieden ausgeprägter, als in den oberen Teilen des Marks. Links verbindet ein ausgedehntes Trümmerfeld, das die Wurzeleintrittszone und die laterale Wurzelzone umfaßt, die Degenerationsgebiete der Hinter- und Seitenstränge, so daß die dort dem Hinterhorn zustrebenden Nervenfasern völlig zerstört sind. Rechts finden sich nur einzelne Zerfallsherde in der Wurzeleintrittszone. Dichter Zerfall ist hingegen in den Py.S.Str. mit massenhaften Zerfallsprodukten besonders in den perivaskulären Lymphräumen, erkennbar. Geringere herdförmige Degenerationen frischer Entstehung zeigen sich in den Py.Vo.Str.

Unteres Brustmark: Die Hinterstrangdegeneration nimmt immer mehr fleckigen Charakter an und rückt hauptsächlich in die Burdachschen Stränge hinein. Laterale Wurzelzone und Wurzeleintrittszone gewinnen auch links wieder normale Beschaffenheit. Die Seiten- und Vorderstrangdegeneration nimmt an Ausdehnung und Dichte ab. Die Zerfallsprodukte sind im Sudanpräparat nicht so reichlich wie weiter oben nachweisbar. Die Ganglienzellen sind noch deutlich verfettet.

Die Glia ist durch das ganze Brustmark hindurch nirgends gewuchert. An Stalle der zerfallenen Nervensubstanz finden sich überall die oben beschriebenen Maschen. Die Gefäßwandungen zeigen bis auf die dichten Zellinfiltrationen, die im unteren Teile viel geringer als weiter oben sind, keine Veränderungen. Nirgends Verschluß von Gefäßlumen, keine Blutaustritte.

Lendenmark: Ausgesprochene fleckig-herdförmige Degeneration in den Hintersträngen: ein größerer mehr dorsal, ein kleinerer mehr ventral gelegener Herd. Dieser enthält reichlich Zerfallsprodulte, dürfte also jüngeren Datums sein, während jener davon vollkommen frei ist. Unverändert geblieben sind die dem Septum mediale im dorsalen Teile anliegenden Gebiete sowie das ventrale und dorsale Hinterstrangfeld und der die graue Substanz seitlich berührende Teil. Unverändert sind also Wurzeleintrittszone, laterale Wurzelzone und Flechsigs ovales Feld.

In den Seitensträngen gleichfalls heidförmige, wenig ausgedehnte, nach unten immer abnehmende Degeneration. Vorderstränge ganz intakt. - Die Gefäße sind überall unverändert, zeigen auch keine zellige Infiltration mehr. 
Das Herz zeigt ausgedehnte Verfettung; Leber, Nieren und Milz ausgeprägte positive Berlinerblaureaktion; in den Nieren findet sich außerdem herdweise Trübung der Harnkanälchenepithelien mit mangelhafter Färbbarkeit der Kerne.

Die beiden eben mitgeteilten Fälle unterscheiden sich sowohl klinisch wie pathologisch-anatomisch in wesentlichen Punkten. Gemeinsam ist beiden Fëllen die Anämie. Während aber in Fall 1 eine solche klinisch nicht nachgewiesen, weil nicht gesucht wurde, und erst der Sektionsbericht die sicheren Anzeichen einer schweren, wenn man will perniziösen Anämie bietet, wurde umgekehrt in Fall 2 hauptsächlich klinisch das einer perniziösen Anämie ähnelnde Bild festgestellt und bei der Seltion zwar die Anzeichen hochgradiger Blutarmut, nicht aber die für schwere Anämie diagnostisch verwertbaren eindeutigen Merkmale gefunden. Im klinischen Bilde selbst spricht manches gegen das Bestehen einer perniziösen Anämie. Jedenfalls ist der niedrice Färbeindex von 0,9, der relativ hohe Hämoglobinwert von $45 \%$ und das Fehlen von Megaloblasten im Blutbilde auch mit der Diagnose sekundäre Anämie verwertbar. Dieser Umstand bietet wohl für die sich ergebenden ätiologischen Betrachtungen gewisse Schwierigkeiten. Im Grunde genommen ist es ja aber für unsere Untersuchungen von nicht allzu großem Belange, welche Form einer Anämie vorgelegen hat, seit wir durch zahlreiche Beispiele wissen, daß die in Rede stehenden Spinalveränderungen oft nur von leichten Anämien begleitet, ja auch unabhängig von primären Anämien vorkommen. Fall 1 dürfen wir ohne Bedenken zu den ,klassischen" Formen von Spinalveränderungen bei perniziöser Anämie rechnen, ähnlich den von Lichtheim im Jahre 1887 beschriebenen Fällen. In ätiologischer Beziehung gestattet dieser uns auch wieder nur Vermutungen, daß eine unbekannte Noxe Rückenmark und Blut gleichzeitig oder nacheinander geschädigt habe. Auch in Fall 2 kommen wir ïber Vermutungen nicht hinaus. Wäbrend es wohl möglich ist, die schwere chronische Tuberkulose als Ursache für die Anämie anzusehen, kann man jene wohl kaum direlzt für die spinal veränderunger verantwortlich machen. Diese müBten ja sonst bei der Häufigkeit der Tuberkulose auch zah]reicher angetroffen werden. Sehr einleuchtend will es mir deshalb erscheinen, wenn Henneberg für die Entstehung der Spinalveränderungen eine zweite Noxe verantwortlich macht, die das durch Anämie geschädigte Rückenmark zerstört. Diese Noxe kann auch ohne Anämie wirksam sein, hat aber mit der Entstehung der Blutveränderung nichts zu tun. Vielleicht wird es mög- 
lich sein, anknüpfend an die Untersuchungen von Faust und Tallquist (1907) und die Experimente von v. VoB (1897) andererseits, in der Frage der Ätiologie dieser Spinalerkrankungen weiterzukommen.

Bei Betrachtung der Degeneration in pathologisch-anatomischer Hinsicht ergibt sich folgendes: In Fall 1 sind die Hinterstränge in ganzer Länge von fleckig-herdförmigen Degenerationen durchsetzt, die sich hauptsächlich in den mittleren Wurzelzonen der Keilstränge lokalisieren. Die Gollschen Stränge sind fast vollkommen unverändert. In den Seitensträngen finden wir im Halsteil die Kl.H.S.Str. systematisch degeneriert, während die Pyramidenseitenstränge nur unbedeutend verändert sind. In den tieferen Teilen des Marks hingegen gewinnt die Degeneration der Pyramidenstrangbahn an Dichtigkeit und die Kl.H.S.Str. verlieren den Charakter systematischer Degeneration. Diese nimmt herdförmigen Charokter an. Die K1.S.Str, sind also sekundär aufsteigend degeneriert, wahrscheinlich wohl durch; eine Unterbrechung der von den Clarkeschen Säulen kommenden nach den Seitensträngen ziehenden Fasern in dem ausgiebig zerstörten Areal der Py.S.Str., die, von oben nach unten zunehmend, reichlich fleckig herdförmigen Faserausfall zeigen. Das fast vollständige Fehlen von Körnchenzellen und der Nachweis von nur geringen Mengen von Abbauprodukten in den Degenerationsbezirken der Hinter- und Seitenstränge, die darin keinen Unterschied erkennen lassen, charakterisicren den Prozeß als ziemlich alten. Wahrscheinlich ist er im großen und ganzen schor längere Zeit vor dem Tode des Patienten zum Stillstand gekommen. Dagegen vermag auch der Nachweis eines einzigen Herdes frischer Fntstehung im oberen Brustmark nichts zu sagen. Hingegen spricht das Verhalten der GefäBe - nach Non nes Ansicht wenigstens - m. E. für diese Annahme: Diese zeigen nur ganz vereinzelt geringfügige kleinzellige Infiltration der Adventitia. Mit dem Stationärwerden des Prozesses ist es auch zu erklären, daß die Degenerationen der Hinter und Seitenstränge gleichartig erscheinen, ohne es wahrscheinlich zu sein. Vielmehr möchte ich die Veränderungen der Hinterstränge für. jünger halten, als die der Seitenstränge, denn nur dadurch kann ich es mir exklären, daß jene, entgegen den Kl.H.S.Str. keine sekundären Degenerationen aufweisen.

In Fall 2 finden wir ein wesentlich anderes Bild. Die Hinterstrünge sind vom Lendenmark aufwärts in rasch zunehmender Stärke degeneriert, so daß die Degeneration im Halsmark systematischen Charakter trägt. Auch. die K1.H.S.Str. machen dort den Findruck 
ziemlich streng systematischer Degeneration, im Gegensatz zu den Py.S.Str., die ausgeprägt flecliig verändert sind. Weiter abwärts kehrt sich das Verhältnis gerade um. Die Py.Vo.Str. zeigen nur vereinzelte Degenerationsherde. Sowohl die Hinter- wie die Vorderstränge bieten also das Bild herdförmiger Veränderungen, auf Grund deren sekundäre Strangdegeneration eingetreten ist und damit das typische Bild der Spinalveränderungen bei ,funikulärer Myelitis“. Ein aus dem Rahmen des Typischen herausfallender Befund liegt in der ausgedehnten Zerstörung der Wurzelzone im mittleren Brustmark. Er vermag vielleicht m. E。 an einem von Bruns mitgeteilten Fall zu erimnern, wo eine „Lrweichung" fast den ganzen Querschnitt des Brustmarks einnahm. Auffallend ist weiterhin die geringe Beteiligung der.Glia sowohl in Fall 1 wie in Fall 2. Die Verfettung der Ganglienzellen in den Vorderhörnern beweist, daß die Veränderungen auch die graue Substanz nicht rollkommen verschonen; es bleibt die $\mathrm{W}$ ahl, diese Veränderungen als Folgen einer retrograden Degeneration oder analog den übrigen audzufassen.

Die dichte kleinzellige Infiltration und reichliche Umlagerung der Gefäße mit Körnchenzellen, sowie die Anhäufung von Zerfallsmassen in den Gefäßscheiden und Gliamaschen charakterisieren den Prozeß im Gegensatz zu Fall 1 als einen frischen und in Gemeinschaft mit dem Nachweis sekundärer Degenerationen als einen subakuten.

Bieten nun unsere Fälle die Möglichkeit, in der lebhaft umstrittenen Frage von der Pathogenese der Spinalveränderungen Klarheit zu gewinnen? - Im wesentlichen handelt es sich dabei um drei Theorien: die in der Literatur Bekenner wie Leugner gefunden haben. Zunäcbst sei der Ansicht Taylors und Tejchmüllers gedacht, die die Veränderungen auf ausgedehnte Blutungen in die Rückenmarkssubstanz hinein zurückführen wollen. Wie diese Ansicht von den meisten Autoren abgelehnt wurde, so können auch wir für unsere Fälle ihr nicht beiptlichten, weil es uns unwahrecheinlich dünlst, daß die wenigen makroskopisch nachgewiesenen Blutungen - mikroskopisch wurden zufällig keine nachgewiesen - Trsaclie so ausgedehnter Veränderungen sein sollten. Ebensowenig vermögen wir uns der eingangs erwähnten Rothmannschen Theorie arizuschließen; denn die Entstehung der Degenerationen aus einzelnen Heiden ist in beiden Fällen zu dentlich ersichtbar, um darüber hinweg die Annahme einer Systemdegeneration, basierend auf der Veränderung der grauen Substanz, zu rechtfertigen.

Die meisten Anhänger hat die schon von Minnich angedeutete, später von Nonne $u$ a. ausgebaute und verfochtene Gefäßtheorie 
gefunden, zu der sich auch Henneberg (1911), allerdings in wesentlich abgeänderter Eorm, bekennt. Fr will den Degenerationsprozeß insofern von den GefäBen abhängig sein lassen, als die am besten mit Blut versorgten Gebiete (die graue Substanz und der ihr direkt benachbarte Saum der weißen Substanz) der Toxinwirkung und Unterernährung am besten widerstehen können. Die beobachteten Gefäßveränderungen würden damit koordiniert neben die Spinalveränderungen als Folge der Toxinwirkung, nicht als Ursache der Degeneration zu stellen, und diese als akute Parenchymdegeneration anzuseben sein.

Nach der Betrachtung unserer Fälle möchten wir der Auffassung Hennebergs beitreten. Trotz eifrigen Suchens ist es uns nicht gelungen, die von Nonne u. a. beschriebenen isolierten Degenerationsherde in unmittelbarer Nachbarschaft von GefäBen festzustellen, obglejch beide Fälle dazı geeignet erschienen und uns dadurch - für unser Reokachtungsmaterial wenigstens - von der direkten Abhängigkeit der Degenerationen von den Gefäßen im Nonneschen Sinne zu überzeugen.

Dortmund, Säuglingsheim. 